\title{
Population-Based Epidemiology, Malignancy Risk, and Outcome of Primary Sclerosing Cholangitis
}

\author{
Kirsten Boonstra, ${ }^{1}$ Rinse K. Weersma, ${ }^{2}$ Karel J. van Erpecum, ${ }^{3}$ Erik A. Rauws, ${ }^{1}$ B.W. Marcel Spanier, ${ }^{4}$ \\ Alexander C. Poen, ${ }^{5}$ Karin M. van Nieuwkerk, ${ }^{6}$ Joost P. Drenth, ${ }^{7}$ Ben J. Witteman, ${ }^{8}$ Hans A. Tuynman, ${ }^{9}$ \\ Anton H. Naber, ${ }^{10}$ Paul J. Kingma, ${ }^{10}$ Henk R. van Buuren, ${ }^{11}$ Bart van Hoek, ${ }^{12}$ Frank P. Vleggaar, ${ }^{3}$ \\ Nan van Geloven, ${ }^{13}$ Ulrich Beuers, ${ }^{1}$ and Cyriel Y. Ponsioen, ${ }^{1}$ on behalf of the EpiPSCPBC Study Group
}

Extensive population-based studies are much needed to accurately establish epidemiology and disease course in patients with primary sclerosing cholangitis (PSC). We aimed to obtain population-based prevalence and incidence figures, insight in disease course with regard to survival, liver transplantation (LT), and occurrence of malignancies, as well as risk factors thereof. Four independent hospital databases were searched in 44 hospitals in a large geographically defined area of the Netherlands, comprising $50 \%$ of the population. In addition, all PSC patients in the three Dutch liver transplant centers and all inflammatory bowel disease (IBD) patients in the adherence area of a large district hospital were identified. All medical records were reviewed on-site, verifying diagnosis. Five hundred and ninety PSC patients were identified, resulting in an incidence of 0.5 and a point prevalence of 6.0 per 100,000. Median follow up was 92 months. Estimated median survival from diagnosis until LT or PSC-related death in the entire cohort was 21.3 years, as opposed to 13.2 years in the combined transplant centers cohort $(n=422 ; P<0.0001)$. Colorectal carcinoma (CRC) risk was 10-fold increased, as compared to ulcerative colitis controls, and developed at a much younger age (39 years; range, 26-64), compared to IBD controls (59 years; range, 34-73; $P=0.019)$. Colonoscopic surveillance was associated with significantly better outcome. Conclusion: This study exemplifies that, for relatively rare diseases, it is paramount to collect observational data from large, population-based cohorts, because incidence and prevalence rates of PSC are markedly lower and survival much longer than previously reported. The selection of a bias-free, population-based cohort showed a significantly longer survival, compared to the tertiary referral cohort. CRC can develop at an early age, warranting surveillance from time of PSC diagnosis. (HEPATOLOGY 2013;58:2045-2055)

P rimary sclerosing cholangitis (PSC) is an enigmatic cholestatic liver disease affecting the intraand extrahepatic bile ducts. PSC is more common in men than in women $(2: 1)$ and can occur at any age, with a peak incidence around $40 .{ }^{1}$ Common symptoms associated with PSC are jaundice, pruritus, and upper abdominal discomfort, although approximately $40 \%$ of patients are asymptomatic at

Abbreviations: $A I H$, autoimmune hepatitis; $C C A$, cholangiocarcinoma; $C D$, Crohn's disease; $C I$, confidence interval; COX, cyclooxygenase; CRC, colorectal carcinoma; ERC, endoscopic retrograde cholangiography; HR, hazard ratio; IBD, inflammatory bowel disease; LT, liver transplantation; MRC, magnetic resonance cholangiography; NF- $\mathrm{B}$, nuclear factor kappa B; NSAIDs, nonsteroidal anti-inflammatory drugs; PSC, primary sclerosing cholangitis; SD, standard deviation; SIR, standardized incidence ratio; SMR, standardized mortality ratio; UC, ulcerative colitis; UDCA, ursodeoxycholic acid

From the ${ }^{1}$ Department of Gastroenterology and Hepatology, Academic Medical Center, Amsterdam, the Netherlands; ${ }^{2}$ Department of Gastroenterology and Hepatology, University of Groningen and University Medical Center Groningen, Groningen, the Netherlands; ${ }^{3}$ Department of Gastroenterology and Hepatology, University Medical Center Utrecht, Utrecht, the Netherlands; ${ }^{4}$ Department of Gastroenterology and Hepatology, Rijnstate Hospital, Arnhem, the Netherlands; ${ }^{5}$ Department of Gastroenterology and Hepatology, Isala Clinics, Zwolle, the Netherlands; ${ }^{6}$ Department of Gastroenterology and Hepatology, VU Medical Center, Amsterdam, the Netherlands; 'Department of Gastroenterology and Hepatology, Radboud University Nijmegen Medical Center, Nijmegen, the Netherlands; ${ }^{8}$ Department of Gastroenterology and Hepatology, Gelderse Vallei Hospital, Ede, the Netherlands; ${ }^{9}$ Department of Gastroenterology and Hepatology, Medical Center Alkmaar, Alkmaar, the Netherlands; ${ }^{10}$ Department of Gastroenterology and Hepatology, Tergooiziekenhuizen, Hilversum/Blaricum, the Netherlands; ${ }^{11}$ Department of Gastroenterology and Hepatology, Erasmus University Medical Center, Rotterdam, the Netherlands; ${ }^{12}$ Department of Gastroenterology and Hepatology, Leiden University Medical Center, Leiden, the Netherlands; ${ }^{13}$ Clinical Research Unit, Academic Medical Center, Amsterdam, the Netherlands.

Received February 5, 2013; accepted May 27, 2013.

This research was supported by unrestricted grants from AbbVie Inc. (Hoofddorp, the Netherlands), Tramedico Inc. (Weesp the Netherlands), and Dr. Falk Pharma Benelux Inc. (Breda, Netherlands). R.K.W. is supported by a clinical fellowship grant (90.700.281) from the Netherlands Organization for Scientific Research (NWO). 
diagnosis. ${ }^{2}$ Multifocal strictures and dilatations of the intra- and/or extrahepatic bile ducts noted on cholangiography are hallmarks of PSC. ${ }^{3}$ PSC is strongly associated with inflammatory bowel diseases (IBDs), and patients are at increased risk for developing colorectal and biliary malignancies. ${ }^{4,5}$ Disease course is highly variable and there is no treatment available with proven efficacy in halting disease progression other than liver transplantation (LT). In Europe, PSC accounts for $9 \%$ of LT indications. ${ }^{6}$ Median survival time until death or liver transplantation is reported to be 12 years. $^{2,7-9}$

Most epidemiological studies on PSC are retrospective case series mainly describing disease course and the association with IBD based on tertiary referral series with their immanent selection bias. Large, population-based data on incidence and prevalence as well as on the natural history of PSC are scarce, but very important for proper patient counseling, finding clues to etiology, as well as for healthcare officials dealing with the planning of future budgets for LT, which is currently one of the most expensive treatments available, averaging $\$ 120,000$.

Here, we describe the largest comprehensive PSC cohort to date to obtain proper population-based prevalence and incidence figures, insight in disease course with regard to survival, need for LT, and occurrence of PSC-related malignancies, as well as risk factors thereof.

\section{Patients and Methods}

Study Design and Participants. The protocol was approved by the central Committee for Research Ethics in Utrecht and all 44 local ethics committees of the participating hospitals in the Netherlands (trialregister.nl no.: NTR2813). An observational longitudinal cohort study was undertaken between January 1, 2008 and Decemeber 31, 2011. All PSC patients in 44 hospitals from 2000 onward were identified in a geographically defined area of six adjacent provinces comprising $50 \%$ of the Dutch population. These 44 hospitals provided care to the entire population of the study area. PSC patients in the three liver transplant centers in the Netherlands (University Medical Center Groningen, Erasmus Medical Center Rotterdam, and Leiden University Medical Center) and all IBD patients without PSC in the adherence area of a large district hospital (Tergooiziekenhuizen) served as control cohorts. $5,10,11$

Case Finding and Case Ascertainment. Case finding was performed according to the guidelines of Metcalf and James. ${ }^{12}$ Four independent hospital databases were searched: (1) PALGA, a nationwide network and comprehensive registry of histo- and cytopathology in the Netherlands, ${ }^{13}$ using diagnosis code liver*biopsy*primary sclerosing cholangitis; (2) hospital billing system using codes 707 (primary sclerosing cholangitis/ primary biliary cirrhosis/autoimmune hepatitis), 954 (primary sclerosing cholangitis/primary biliary cirrhosis) and 943 (autoimmune hepatitis) (International Classification of Diseases, Tenth Revision code K83.0); (3) endoscopic retrograde cholangiography (ERC) reports in endoscopy-suite databases; and (4) personal lists of treating physicians. IBD patients were searched on-site using the same four data sources. PSC registries in the three liver transplant centers in the Netherlands and the IBD registry in the pertaining affiliated large tertiary referral center were checked for missed referrals from the area of interest. All medical records were scrutinized on-site by two investigators (K.B. and C.P.) for ascertainment of PSC diagnosis. Diagnosis was based on the following: (1) clinical presentation (i.e., pruritus), pain in the right upper abdominal quadrant, fatigue, weight loss, and episodes of fever; and/or (2) elevated alkaline phosphatase and gamma-glutamyl transferase, not otherwise explained; (3) presence of characteristic bile duct changes with multifocal strictures and segmental dilatations on ERC or magnetic resonance cholangiography (MRC); and/or (4) liver histology; and (5) no evidence for secondary sclerosing cholangitis. When criteria 2, 4, and 5 were fulfilled in the absence of cholangiographic abnormalities on MRC or ERC, cases were diagnosed as small-duct PSC. ${ }^{14}$ Autoimmune hepatitis (AIH) overlap syndrome (PSC$\mathrm{AIH}$ ) is ill defined. A diagnosis of PSC-AIH was made in

Address reprint requests to: Cyriel Y. Ponsioen, M.D., Ph.D., Department of Gastroenterology and Hepatology, Academic Medical Center, P.O. Box 22700,

1100 DE Amsterdam, the Netherlands. E-mail: c.y.ponsioen@amc.uva.nl; fax: +31 206917033.

Copyright $(\mathcal{C} 2013$ by the American Association for the Study of Liver Diseases.

View this article online at wileyonlinelibrary.com.

DOI 10.1002/hep.26565

Potential conflict of interest: Dr. Beuers consults for Intercept and Novartis. He received lecture fees from the Falk Foundation, Roche, Gilead, and Zambon. Dr.

Ponsioen received grants from AbbVie, Falk, and Tramedico.

Additional Supporting Information may be found in the online version of this article. 
patients with a characteristic cholangiogram who, in addition, met the simplified AIH criteria. ${ }^{15}$ IBD diagnosis was based on the Lennard-Jones criteria. ${ }^{16}$

Data Collection. At study inclusion and during follow-up, apart from demographic characteristics, the following data and endpoints were collected: date and type of PSC diagnosis; date of diagnosis and type of IBD, including Montreal classification; date and cause of death; date and type of malignancy; date and type of surgery; and cumulative medication use $\geq 6$ months for ursodeoxycholic acid (UDCA), thiopurines, and mesalamine during follow-up. For nonsteroidal antiinflammatory drugs (NSAIDs), including aspirin, any prescription use was counted. Date of PSC diagnosis is defined as the date of the diagnostic procedure confirming the diagnosis (ERC, MRC, or liver biopsy). Colonoscopic surveillance was defined as a full colonoscopy at PSC diagnosis and every 1-2 years after diagnosis in PSC-IBD patients. Occurrence of carcinoma in the cohort was double checked by linkage of the PSC cohort to PALGA, the nationwide network and registry of histo- and cytopathology in the Netherlands. ${ }^{13}$

Annual follow-up was obtained by written correspondence from the treating physician. Date of diagnosis was the starting point of follow-up. End of follow-up was defined as death, last visit to outpatient clinic, or end of the study (January 2012). PSC-related death was defined as death caused by liver disease, cholangiocarcinoma (CCA) or CRC. Date of death was retrieved from the national death registry. Mid-year, age, and sexspecific population estimates were based on data from the Dutch Central Office for Statistics (http://statline.cbs.nl/statweb/). Data on the incidence of malignancies in the general population were retrieved from the Dutch Cancer Registry (www.cijfersoverkanker.nl).

Statistical Analyses. Date of diagnosis was defined as the starting point of the disease for all analyses. Cochrane-Armitage test for trend was used to test changes in prevalence and incidence. Kaplan-Meier's survival analysis was performed to estimate cumulative survival. Estimated median survival times were calculated for (1) combined endpoint PSC-related death or LT, (2) LT, and (3) PSC-related death censored at LT. Overall difference in survival was investigated by the log-rank test. Standardized mortality ratio (SMR) and standardized incidence ratio (SIR) were calculated as the ratios of observed, compared with expected, number of deaths and malignancies in the study cohort. The expected number of patients was calculated based on age- and gender-specific mortality and malignancy rates in the general population. When calculating cumulative risk and SIR for CCA, censoring was performed for LT. In the case of dysplasia or CRC, cases were censored at time of colectomy. The extended Cox model for time-dependent (CCA, CRC, LT, and colectomy) and -independent variables (age at diagnosis, gender, PSC type, AIH, IBD, extension colitis, and medication) was used for uni- and multivariate analysis of risk factors for endpoints PSC-related death, LT, CCA, and CRC. The assumption of proportional hazards was tested using $\log$ minus log survival plots and found valid. Risk factors with a $P$-value $<0.1$ in univariate analysis were entered in the multivariate analysis. MannWhitney's U-test was performed for comparing continuous data with a non-normal distribution. The chi-square test or Fisher's exact test were used for categorical data. Statistical analyses were performed using SPSS v. 19.0 software (SPSS, Inc., Chicago, IL). $P<0.05$ was considered statistically significant.

\section{Results}

Study Inclusion. The case-finding strategy yielded 3,020 individuals. Of these, 695 were alive on January 1, 2000 and fulfilled the diagnostic criteria for PSC. Reasons for study exclusion are depicted in Fig. 1. Five hundred and ninety PSC patients were resident in the predefined study area between 2000 and 2007 (Supporting Fig. 1). The total number of inhabitants increased during the study period from 7,342,295 in 2000 to $7,758,980$ in 2007 . The second PSC cohort, accrued from the three Dutch transplantation centers outside the study region, yielded 450 cases, of whom 134 (30\%) were also present in the population-based cohort. ${ }^{5,10,11}$ Of these, 422 were alive after January 1, 2000 and entered in the comparison. The IBD control cohort comprised 722 cases from a population of 271,000.

Patient Characteristics. Main patient characteristics are shown in Table 1. Median follow-up from diagnosis until death or end of study was 92 months (range, 0-470). Mean age at diagnosis was 38.9 (standard deviation [SD]: 15.2) years. Among the 590 patients, 375 (64\%) were male. Fifty-eight (9\%) patients had small-duct PSC and 23 (4\%) fulfilled the criteria for overlap syndrome with AIH. In total, 402 (68\%) PSC patients were diagnosed with IBD. Overall, 393 (98\%) PSC-IBD patients had ulcerative colitis (UC) or colonic Crohn's disease (CD) and only 5 of $78(6 \%)$ CD patients had isolated ileal disease. Ninety-two percent (493 of 534) of patients were treated with UDCA, 68\% (300 of 438) with mesalamine, $35 \%$ (118 of 333 ) with thiopurines, and $34 \%$ (107 of 317) of patients were using NSAIDs. 


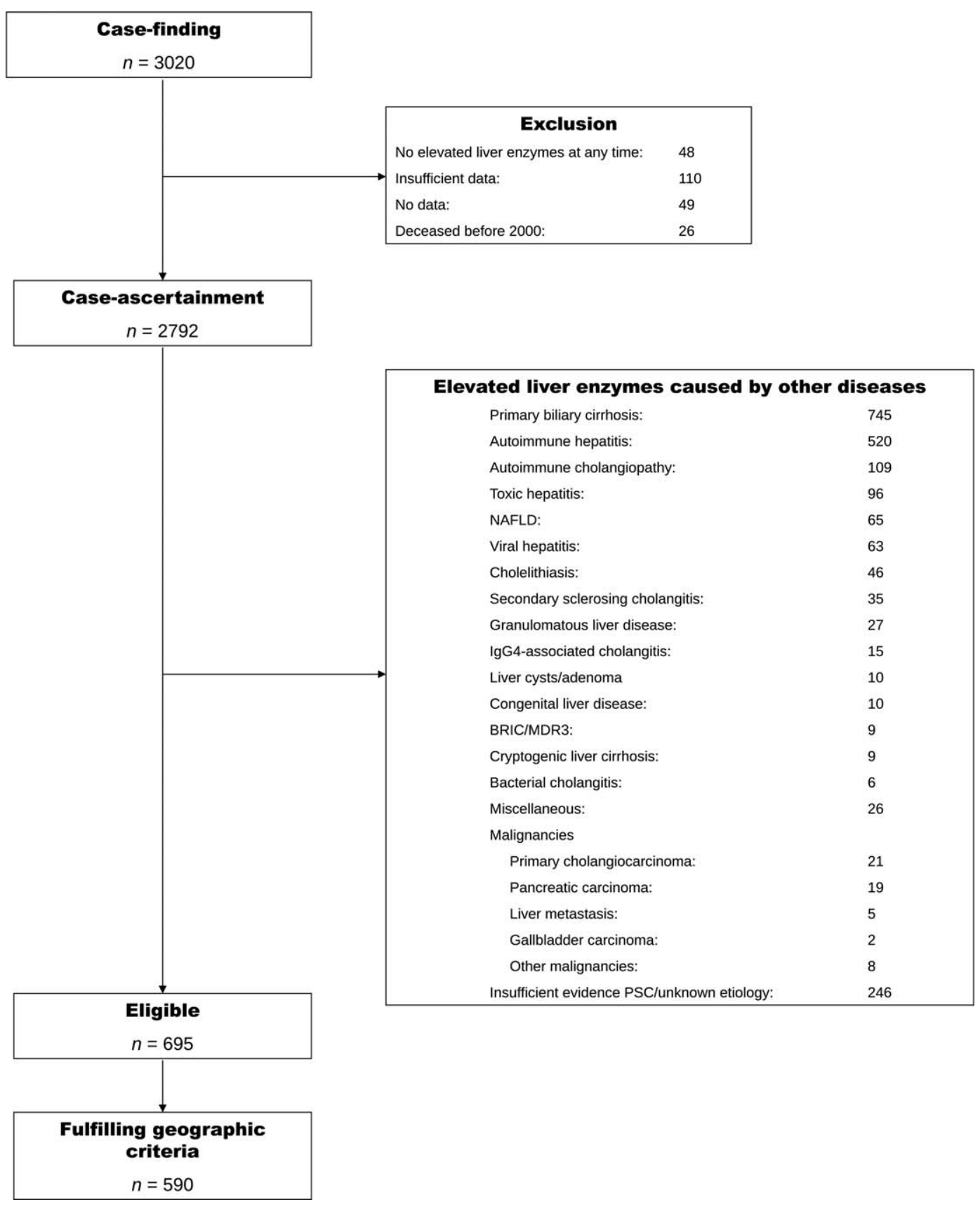

Fig. 1. Flowchart patient inclusion.

Incidence and Prevalence. Mean annual incidence between 2000 and 2007 was 0.5 per 100,000 inhabitants (0.6 in men and 0.4 in women). Age- and gender-standardized incidence rates ranged from 0.25 in female adolescents up to 0.93 per 100,000 in men between the ages of 40 and 49 (Fig. 2A). On January 1, 2008, point prevalence was 6.0 per 100,000 inhabitants; prevalence rates increased significantly over time $(P<0.001$; Fig. $2 \mathrm{~B})$. Net growth in prevalence was attributable to increase in incidence and not to a decrease in number of deaths (data not shown). In addition, bilirubin levels at diagnosis did not change between 2000 and 2007 (Supporting Fig. 2).

Natural History. During follow-up, there were 97 deaths, of which 73 (75\%) were PSC related. Sixteen (3\%) patients were lost to follow-up. Estimated median survival times from diagnosis until the combined endpoint LT $(\mathrm{n}=94)$ or PSC-related death ( $\mathrm{n}$ $=73$ ), until LT, or until PSC-related death were 21.2, 27.0, and 33.6 years, respectively. When including all deaths in the analysis, the estimated median 
Table 1. Patient Characteristics

\begin{tabular}{|c|c|c|c|c|c|}
\hline Characteristics & $\begin{array}{l}\text { Population-Based } \\
\text { PSC Cohort n (\%) }\end{array}$ & $\begin{array}{l}\text { Liver Transplant Centers } \\
\text { PSC Cohort n (\%) }\end{array}$ & $P$ Value & IBD Cohort n (\%) & $P$ Value \\
\hline No. of patients & 590 & 450 & & 722 & \\
\hline Male & $375(64)$ & $291(65)$ & 0.710 & $310(43)$ & $<0.001$ \\
\hline Age at PSC diagnosis, years (mean [SD]) & $38.9(15.2)$ & $36.2(13.8)$ & 0.004 & NA & \\
\hline AlH overlap & $23(4)$ & $47(10)$ & $<0.001$ & NA & \\
\hline Follow-up (months) (median [range]) & $92(0-470)$ & $80(0-354)$ & $<0.001$ & $98(0-584)$ & 0.010 \\
\hline IBD & $402(68)$ & $287(64)$ & 0.140 & & \\
\hline Ulcerative colitis & $308(77)$ & $224(78)$ & 0.020 & $429(59)$ & $<0.001$ \\
\hline Crohn's disease & $78(19)$ & $40(14)$ & & $208(29)$ & \\
\hline Unspecified & $16(4)$ & $23(8)$ & & $85(12)$ & \\
\hline Age at IBD diagnosis, years (mean [SD]) & $31.5(15.1)$ & $30.2(14.0)$ & 0.290 & $41.2(16.9)$ & $<0.001$ \\
\hline
\end{tabular}

Abbreviation: NA, not available.

transplant-free survival was 20.6 years. Because survival was considerably longer than previously reported, a second cohort was accrued consisting of 450 PSC
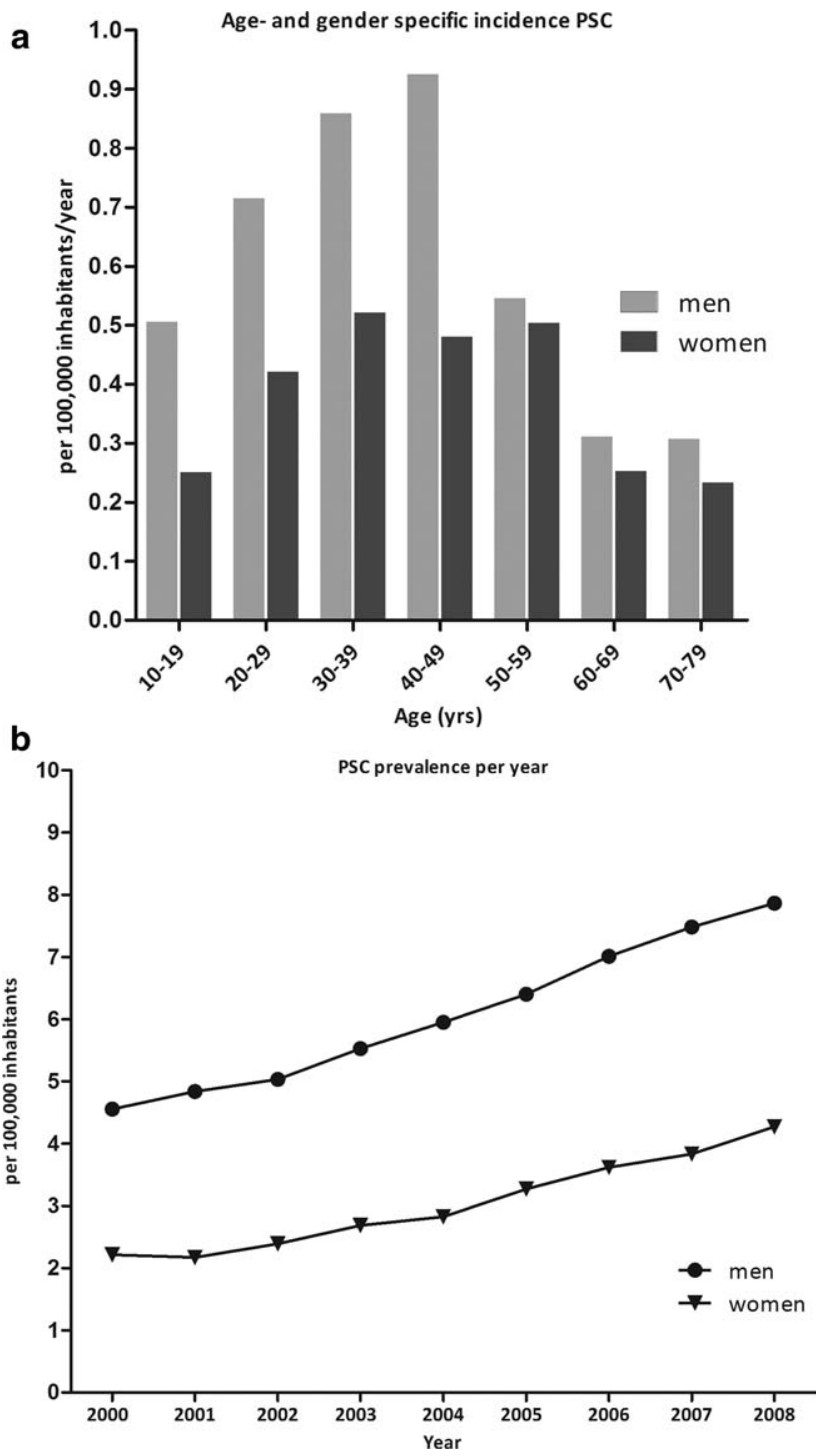

Fig. 2. Age- and gender-specific incidence of PSC (A) and point prevalence of PSC per 100,000 inhabitants per year (B) in a population of $7,758,980$. Net growth in prevalence was highly significant $(P<0.001)$. patients from all three liver transplant centers in the Netherlands to assess the influence of referral bias. Notably, the population-based cohort showed a significantly longer survival from date of diagnosis until the combined endpoint LT or PSC-related death, compared to this tertiary referral centers cohort $(21.3$ versus 13.2 years; $P<0.0001$; Fig. 3 ).

Patients with small-duct PSC had a much better survival until PSC-related death or LT, compared with large-duct PSC ( $P=0.019$; Supporting Fig. 3). Concurrence of AIH did not affect transplant-free survival $(P=0.58)$. PSC patients had a 4 -fold increased risk of mortality, compared to the general population (SMR, 4.2; 95\% confidence interval [CI]: 3.2-5.4). The four most-frequent causes of death were CCA (32\%), liver failure (18\%), OLT-related complications (9\%), and CRC (8\%). Ninety-four patients received a liver transplant after median disease duration of 8.1 years (range, 0.3-31.3). In multivariate analysis, age at diagnosis, CRC, and CCA were risk factors for endpoint PSC-related death. NSAID use was associated

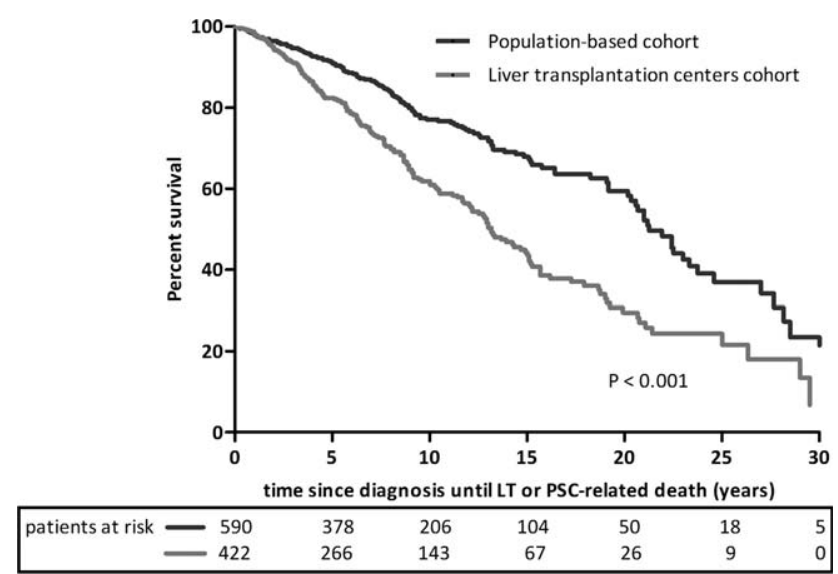

Fig. 3. Survival until LT or PSC-related death of PSC patients in the population-based cohort, compared to PSC patients, referred to LT centers. 
Table 2. Univariate and Multivariate Analyses for Endpoints PSC-Related Death and LT

\begin{tabular}{|c|c|c|c|c|c|c|}
\hline \multirow[b]{2}{*}{ Endpoint PSC-Related Death } & \multicolumn{3}{|c|}{ Univariate Analysis } & \multicolumn{3}{|c|}{ Multivariate Analysis } \\
\hline & HR & $95 \% \mathrm{Cl}$ & $P$ Value & HR & $95 \% \mathrm{Cl}$ & $P$ Value \\
\hline Age at diagnosis & 1.05 & $1.03-1.06$ & $<0.001$ & 1.06 & $1.02-1.10$ & 0.005 \\
\hline Female gender & 1.62 & $1.01-2.60$ & 0.046 & 0.62 & $0.25-1.54$ & 0.300 \\
\hline Small-duct PSC & 0.12 & $0.03-1.44$ & 0.110 & Not in model & & \\
\hline Concurrence of AlH & 0.34 & $0.05-2.50$ & 0.290 & Not in model & & \\
\hline Concurrence of IBD & 1.16 & $0.68-1.98$ & 0.590 & Not in model & & \\
\hline Extension of colitis & 1.43 & $0.62-3.30$ & 0.400 & Not in model & & \\
\hline $\mathrm{CCA}$ & 9.51 & $5.88-15.40$ & $<0.001$ & 8.84 & 3.63-21.54 & $<0.001$ \\
\hline CRC & 3.09 & $1.55-6.14$ & 0.001 & 4.26 & $0.97-18.97$ & 0.056 \\
\hline LT & 0.60 & $0.33-1.08$ & 0.087 & 1.02 & $0.44-2.34$ & 0.970 \\
\hline Colectomy & 2.04 & $1.15-3.62$ & 0.014 & 1.19 & $0.35-4.06$ & 0.790 \\
\hline UDCA & 1.37 & $0.59-3.18$ & 0.460 & Not in model & & \\
\hline 5-ASA & 1.30 & $0.68-2.49$ & 0.430 & Not in model & & \\
\hline Thiopurines & 0.41 & $0.18-0.94$ & 0.036 & 0.82 & $0.32-2.12$ & 0.680 \\
\hline NSAID & 0.28 & $0.08-0.91$ & 0.035 & 0.28 & $0.06-1.33$ & 0.110 \\
\hline \multicolumn{7}{|c|}{ Endpoint Liver Transplantation } \\
\hline Age at diagnosis & 1.00 & $0.99-1.02$ & 0.670 & 1.01 & $1.00-1.03$ & 0.420 \\
\hline Female gender & 0.71 & $0.45-1.11$ & 0.140 & 0.57 & $0.30-1.06$ & 0.074 \\
\hline Small-duct PSC & 0.28 & $0.07-1.13$ & 0.074 & 0.56 & $0.14-2.36$ & 0.430 \\
\hline Concurrence of AlH & 1.46 & $0.59-3.64$ & 0.410 & Not in model & & \\
\hline Concurrence of IBD & 1.14 & $0.71-1.83$ & 0.580 & Not in model & & \\
\hline Extension of colitis & 1.43 & $0.73-2.78$ & 0.290 & Not in model & & \\
\hline Colectomy & 1.22 & $0.68-2.20$ & 0.510 & Not in model & & \\
\hline UDCA & 1.60 & $0.74-3.46$ & 0.240 & Not in model & & \\
\hline 5-ASA & 0.60 & $0.38-0.95$ & 0.029 & 0.77 & $0.55-1.28$ & 0.310 \\
\hline NSAID & 0.44 & $0.23-0.85$ & 0.014 & 0.46 & $0.22-0.94$ & 0.033 \\
\hline
\end{tabular}

The extended Cox model for time-dependent and -independent variables was used for uni- and multivariate analysis of risk factors for endpoints PSC-related death, LT, CCA, and CRC. Risk factors with a $P$-value $<0.1$ in univariate analysis were entered in the multivariate analysis.

Abbreviation: 5-ASA, 5-aminosalicylic acid.

with a decreased risk for the endpoint LT $(\mathrm{P}=0.018$; Table 2).

CCA occurred in 41 of 590 (7\%) patients, of whom 33 died $(80 \%)$ after a median period of 1 year (range, 0-7). Median age at CCA diagnosis was 47 years (range, 21-87), and median time between PSC diagnosis and CCA was 6 years (range, 0-36). All patients except one had large duct PSC and 27 (66\%) had concomitant IBD, mainly UC. There was a 398fold increased risk for developing CCA in PSC patients compared, to the general population (SIR, 398; 95\% CI: 246-608). Five (12\%) patients were diagnosed with PSC and CCA at initial presentation, another $6(15 \%)$ within the first year, $15(37 \%)$ between 1 and 10 years, and the remaining 15 (37\%) developed a CCA 10 or more years after PSC diagnosis. Cumulative risk of CCA after 10, 20, and 30 years was 6\% (95\% CI: 2-15), 14\% (95\% CI: 4-25), and 20\% (95\% CI: 6-40), respectively (Fig. 4A). Older age at PSC diagnosis was an independent risk factor for endpoint CCA (hazard ratio [HR]: 1.02; 95\% CI: 1.00-1.04; $P=0.049$ ). Occurrence of $\mathrm{CRC}$ was a time-dependent risk factor for CCA (HR, 4.57; 95\% CI: $1.08-19.41 ; P=0.040)$.
Twenty of five hundred and ninety PSC patients (3\%) developed CRC, of whom 10 died (8 CRC related and 2 CCA related). Median age at CRC diagnosis was 41 years (range, 26-64; Fig. 5). Cumulative risk of high-grade dysplasia or CRC after 10, 20, and 30 years since PSC diagnosis was 3\%, 7\%, and 13\%, respectively (Fig. 4A). All CRC patients had large-duct PSC. There was a 5-fold increased risk for developing CRC in all PSC patients, compared to the age- and gender-matched general population (SIR, 5.0; 95\% CI: 2.02-10.3). IBD was present in 19 (95\%) patients (18 UC and $1 \mathrm{CD}$ ), and median time between IBD diagnosis and CRC was 15 years (range, 0-35). One CRC patient had no endoscopic or histologic signs of IBD after 14 years of follow-up. Seven (7/722) IBD control patients developed CRC (SIR, 1.2; 95\% CI: 0.3-3.0) after a median time span of 4 years (range, 0-19) after diagnosis (4 UC, 1 CD, and 1 IBD unspecified). There was a 9-fold increased risk for developing CRC in PSC-UC patients, compared to the ageand gender-matched population (SIR, 8.6; 95\% CI: 3.5-17.7), and a 10-fold increased risk, compared to UC controls (ratio of SIRs: 9.8; 95\% CI: 1.9-96.6). Strikingly, CRC occurred at a much younger age in 

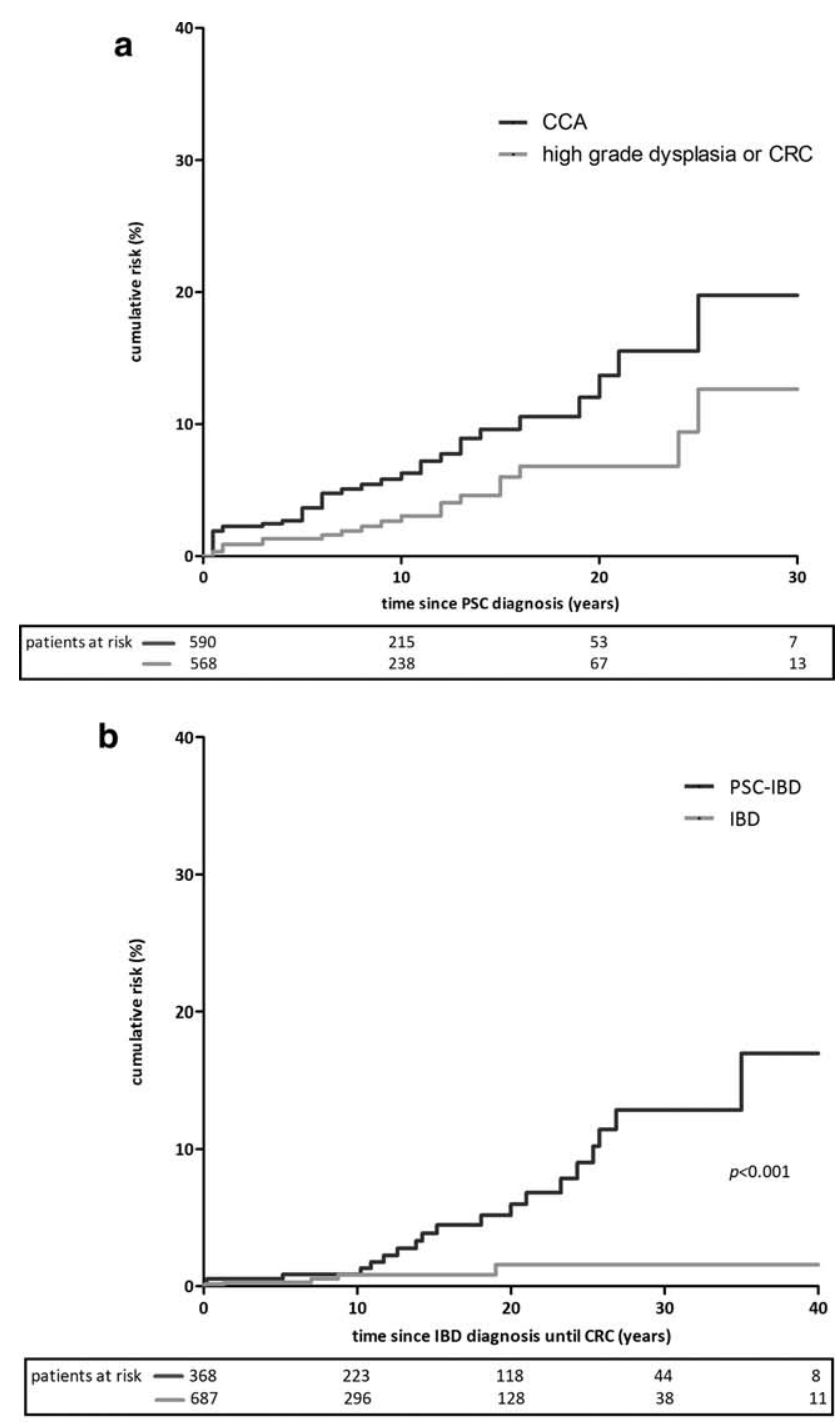

C

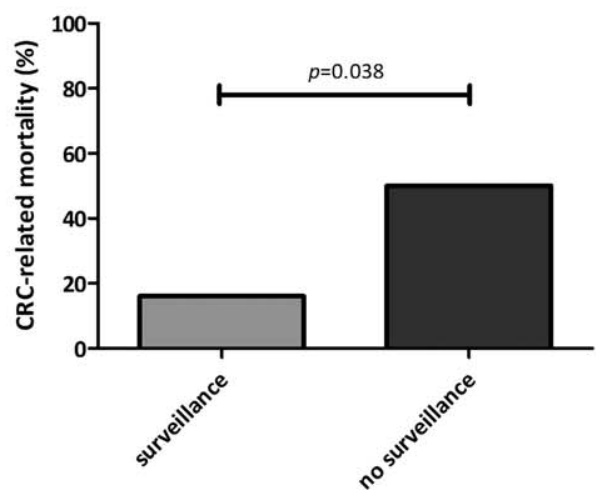

Fig. 4. Malignancy risk in PSC patients and controls. (A) Cumulative risk of CCA and high-grade colon dysplasia or CRC in PSC patients. Twenty-two patients with proctocolectomy or CRC before diagnosis of PSC were excluded from the analysis. (B) Cumulative risk of CRC in PSC-IBD patients and IBD controls in whom onset of IBD was known. $C D$ patients with isolated ileitis were excluded from cumulative risk analysis for CRC. (C) CRC-related mortality in surveilled and nonsurveilled PSC-IBD patients.
PSC-IBD patients (median age: 39; range, 26-64), compared to IBD controls (59; range, 34-73; $P=$ 0.019). For PSC-IBD patients, cumulative risk of CRC after 10, 20, and 30 years since IBD diagnosis was 1\% (95\% CI: 0-15), 6\% (95\% CI: 1-22), and 13\% (95\% CI: 2-37), respectively (Fig. 4B). Surveillance colonoscopies had been performed before CRC diagnosis in $53 \%$ of PSC-IBD patients with CRC. When combining PSC-CRC patients from the population-based and transplantation centers cohorts, $50 \%$ (9 of 18) of the nonsurveilled patients and $16 \%$ (3 of 19) who received regular surveillance colonoscopies died of CRC ( $P=0.038$; Fig. 4C).

\section{Discussion}

Here, we present, by far, the largest population-based study of PSC with the longest follow-up to date. We report markedly lower incidence and prevalence rates of 0.5 per 100,000 and 6.0 per 100,000 inhabitants, respectively, compared to previous studies. ${ }^{17-20}$ Prevalence is rising with stable mortality over time (data not shown), which is in concordance with a recent systematic review and perhaps explained by the rising incidence of IBD in North-West Europe. ${ }^{17,21,22}$ Increased incidence could not be explained by earlier diagnosis, because bilirubin levels at diagnosis remained stable over time and newer diagnostic modalities were not introduced in the observed period. The difference with previous studies points to the importance of performing large, population-based studies in relative rare diseases to obtain accurate observational data.

Most epidemiological studies on PSC have been performed in specialized centers, prone for selection and referral bias. Between 1984 and 2012, only five small high-quality population-based studies have been performed reporting incidence rates, ranging from 0 to 1.6 per 100,000 inhabitants, based on a collective number of only 96 PSC patients. ${ }^{17-20,23,24}$ When studying a rare disease in only a few hundred thousand, two or three cases more or less can make a huge difference on incidence and prevalence rates. In contrast, the present results are based on 590 carefully ascertained PSC patients in all hospitals in a geographically defined area of almost 8 million inhabitants. Moreover, by using four different case-finding searches in each center, we have done our utmost to recruit every single PSC patient. As an additional case-finding exercise, all referrals to the three liver transplant centers in the Netherlands were checked for area of residence. This resulted in 52 additional patients. 


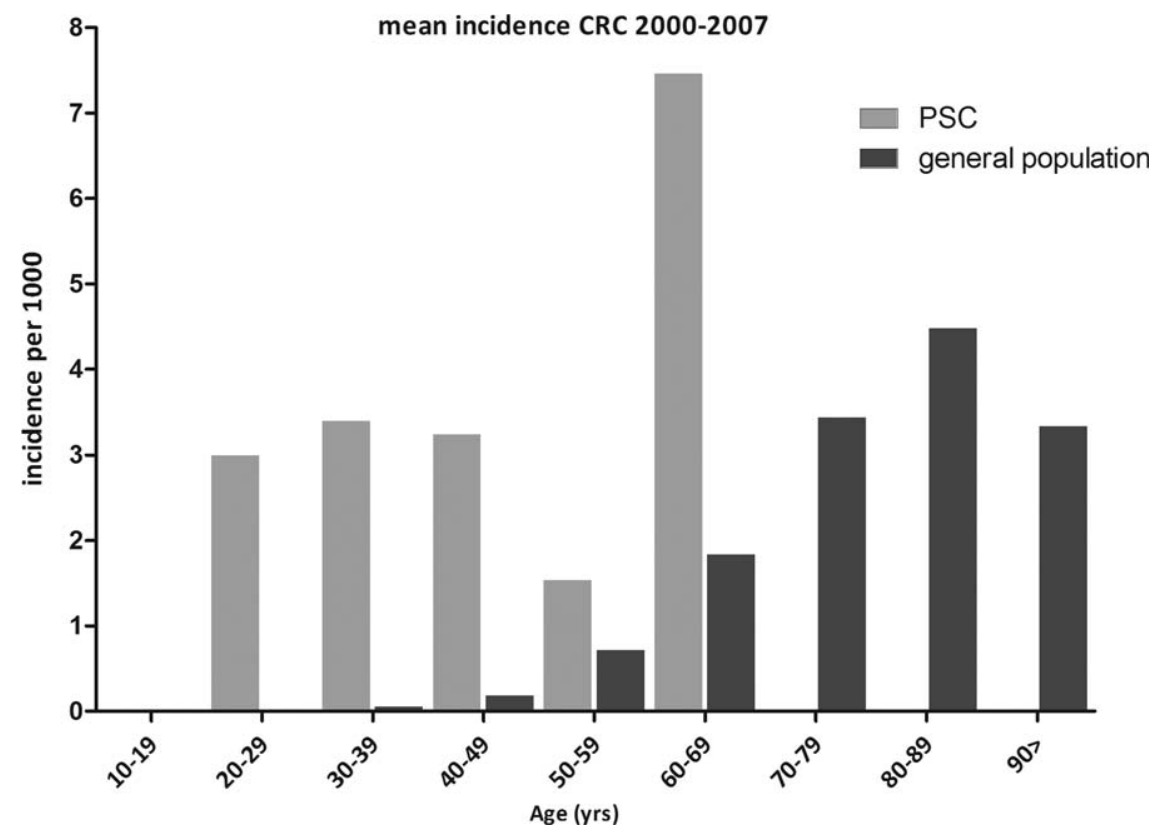

Fig. 5. Mean incidence of colorectal carcinoma in PSC patients and the Dutch population.

Estimated median survival until LT or PSC-related death found in the present study is much longer, compared to previous reports. When comparing studies on the natural history of PSC, several factors play an important role in survival analysis: starting point of disease; definition of endpoints; and proportion of patients that underwent LT. Introduction of MRC as a noninvasive diagnostic tool, physician awareness, and an increase in routine laboratory blood tests may have resulted in an earlier detection of the disease over time. The vast majority of investigators use time of diagnosis as the starting point for follow-up. ${ }^{1,2,5,8,9}$ Definition of PSC-related death differs between studies. Some studies used death from any cause as endpoint for Kaplan-Meier's analysis. ${ }^{5,7,9,10,25}$ When we included all deaths in the analysis, the estimated median survival until LT or death was 20.6 years. In three of the largest studies, death from liver disease was used as an endpoint, excluding death from CRC. ${ }^{1,2,8}$ Considering the increased risk for CRC in PSC, we included CRC-related death in the survival analysis. However, when the 8 CRC-related deaths were excluded from analysis, estimated median survival was 21.3 years, a difference of only 0.1 year. Median transplant-free survival estimates have been reported in the last 25 years ranging from 9.3 years up to 18. ${ }^{1,2,5,7-10,25}$ However, these figures were all based on cohorts from large referral or LT centers, which may constitute another important bias. As in our cohort, in all but one of these studies the starting point for follow-up was date of diagnosis. In a recent singlecenter cohort from Germany reporting a transplantfree median survival of 9.3 years, $40 \%$ of patients had undergone LT versus $16 \%$ in our cohort. ${ }^{9}$ In the present study, we demonstrate the effect of selection bias by comparing our general population-based cohort with a cohort comprising all PSC patients, matched for the population-based cohort inclusion criteria, alive after January 1, 2000, as well as $>=18$ years at inclusion $(n=422)$ from the three transplantation centers in the Netherlands. This contemporary tertiary referral cohort, which had a $30 \%$ overlap with the populationbased cohort, but immanent by its referral nature not matched for baseline characteristics (Table 1), showed an estimated median survival until LT or PSC-related death of 13.2 years. This figure is in concordance with the literature, but almost $40 \%$ shorter than patients included in our population-based cohort. These findings highlight the influence of selection bias and hence again the importance of performing large, populationbased studies to get a reliable estimate of the natural history of a disease.

The high preponderance of UDCA treatment in our cohort precluded investigating the influence of this drug on disease course. However, UDCA was also routinely prescribed to most patients in the transplant centers cohort. Moreover, the three largest randomized studies have not been able to demonstrate a benefit of UDCA in halting disease progression, although this does not exclude an effect in early-stage PSC. ${ }^{26-28}$ 
The present study describes, for the first time, a negative association between NSAID use and LT. Most NSAIDs inhibit cyclooxygenase (COX), an enzyme that converts arachidonic acid into prostaglandin. There are some reports that COX-2 is overexpressed in liver cirrhosis from hepatitis B virus and hepatitis C virus, as well as in various malignancies, including HCC. ${ }^{29,30}$ Interestingly, Chávez et al..$^{31}$ showed a beneficial effect of acetyl salicylic acid and ibuprofen in an experimental rat model of liver fibrosis. The NSAIDs used in this model inhibited oxidative stress, COX-2 activity and nuclear factor kappa B (NF- $\kappa \mathrm{B})$ translocation to the nucleus. NF- $\kappa \mathrm{B}$ plays a central role in the inflammation-fibrosis-cancer axis in the liver by inducing tumor necrosis factor alpha and interleukin-6 activating hepatic stellate cells to produce profibrogenic factors. ${ }^{32}$ Recently, a large, prospective study showed that NSAIDs reduced the risk of death resulting from chronic liver disease, even in individuals who only used NSAIDs less than two to three times per month. ${ }^{33}$ Our finding clearly deserves further investigation.

Patients with PSC are at highly increased risk for developing CCA, which has a very dismal prognosis. Developing surveillance tools for CCA is one of the prime unmet needs in PSC. CCA was diagnosed within the first year after diagnosis in $2 \%$ of PSC patients. Thereafter, the cumulative risk of developing CCA gradually rose to an estimated $20 \%$ after 30 years, arguing against a distinct procarcinogenic subphenotype with a high incidence of early CCA. ${ }^{25,34,35}$ Moreover, it is well known that PSC in retrospect can have a long subclinical time lag until diagnosis of up to 38 years (median, 4.3 ). ${ }^{2}$

CRC is reported to occur more frequently in IBD patients than in the general population. ${ }^{36}$ We observed that the CRC risk in a population-based IBD cohort is equal to the general population. In accord with our findings, a study from Denmark showed that CRC risk in UC patients has declined in the last 30 years and no longer exceeds that of the general population. However, this excluded UC patients diagnosed at a young age, with a long disease duration or concomitant PSC. ${ }^{37}$ PSC is an additional and independent risk factor for development of CRC in UC patients, although low absolute numbers often hamper standardized incidence ratio analysis, emphasizing the need for large, population-based cohorts. ${ }^{38-40}$ Our study confirms the increased risk for CRC with a 5-fold increase in all PSC patients, compared to the general population, and a 10fold increase in PSC-UC patients, compared to UC alone. Furthermore, CRC develops, on average, more than 20 years earlier, compared to IBD patients and the general population, and ranks among the top four causes of death in PSC patients, corroborating current guidelines to start surveillance every 1-2 years from start of diagnosis. ${ }^{14,41}$ PSC-IBD patients whose CRC was discovered in a surveillance program showed a significantly better outcome.

Limitations of this study lie in its retrospective nature with its immanent risk of incomplete data sets. However, the proportion of patients lost to follow-up was only $3 \%$, and because we focused on rather easyto-appreciate endpoints, such as LT, death, and occurrence of CCA, we feel that the chance of missing these data is limited. Also, true population-based figures are impossible to obtain because PSC is a hospital diagnosis. Although we have extensively searched all hospitals in the predefined geographic area and the potential tertiary referral hospitals, there is always a chance of missing some cases and therefore slightly underestimating incidence and prevalence. The capture-recapture method, which can correct for this, could not be applied because three of four data sources were local databases. Not all PSC patients without clinical signs of bowel disease have undergone a screening colonoscopy in the past, which may give an underestimation of concurrent subclinical IBD.

In conclusion, for accurate assessment of epidemiology and natural history of uncommon diseases such as PSC, extensive population-based studies adhering to rigorous case-finding guidelines, such as the Metcalf and James criteria, ${ }^{12}$ are indispensable. We found that the incidence and prevalence rates of PSC are markedly lower and survival much longer than previously reported. Notably, the population-based cohort showed a significantly longer survival until LT or PSC-related death, compared to a tertiary referral centers cohort, exemplifying the effect of selection bias. The incidence of cholangiocarcinoma in PSC patients is high and colorectal carcinoma can develop at a young age, warranting endoscopic surveillance from the time of diagnosis.

Acknowledgment: The authors thank all the of following local investigators: E.T. Abilakh Missier, Röpcke-Zweers Hospital, Hardenberg; L.C. Baak, Onze Lieve Vrouwe Gasthuis, Amsterdam; N. van Bentem, Medical Spectrum Twente, Enschede; J.M. van den Brande, Tergooi Hospitals, Hilversum; M.A. Brink, Meander Medical Center, Amersfoort; W. Bruins Slot, Spaarne Hospital, Hoofddorp; D.L. Cahen, Amstelland Hospital, Amstelveen; M.M Claessen, University Medical Center Utrecht, Utrecht; A.C. Depla, Slotervaart Hospital, Amsterdam; G. de Groot, 
Rode Kruis Hospital, Beverwijk; S.J. van den Hazel, Slingeland Hospital, Doetinchem; M. Janse, University Medical Center Groningen, Groningen; I.J. Klompmaker, Wilhelmina Hospital, Assen; K.S Korkmaz, Leiden University Medical Center, Leiden; J.Ph. Kuyvenhoven, Kennemer Gasthuis, Haarlem; M. Ledeboer, Deventer Hospital, Deventer; B.C. Loffeld, Zuwe Hofpoort, Woerden; R.J. Loffeld, Zaans Medical Center, Zaandam; R.C. Mallant-Hent, Flevo Hospital, Almere; P.R. Oosting, Waterland Hospital, Purmerend; S.C. Riemens, Diaconessenhuis, Meppel; J. Schmidt Böhmer, Westfries Gasthuis, Hoorn; P. Scholten, Sint Lucas Andreas Hospital, Amsterdam; P.H. Stadhouders, St. Antonius Hospital, Nieuwegein; G. Tan, Ziekenhuisgroep Twente, Hengelo; A. Teunen, Boven IJ Hospital, Amsterdam; J.C. Thijs, Bethesda Hospital, Hoogeveen; M.A. Verhagen, Diakonessenhuis, Utrecht; P.P. Viergever, Gemini Hospital, Den Helder; A.A. Vrij, Ziekenhuisgroep Twente, Almelo; O. Weinhardt, Scheper Hospital, Emmen; and E.M. Witteman, Gelre Hospitals, Apeldoorn.

\section{References}

1. Ponsioen CY, Vrouenraets SME, Prawirodirdjo W, Rajaram R, Rauws EA, Mulder CJ, et al. Natural history of primary sclerosing cholangitis and prognostic value of cholangiography in a Dutch population. Gut 2002;51:562-566.

2. Broomé U, Olsson R, Löof L, Bodemar G, Hultcrantz R, Danielsson A, et al. Natural history and prognostic factors in 305 Swedish patients with primary sclerosing cholangitis. Gut 1996;38:610-615.

3. Ponsioen CY, Lam K, van Milligen de Wit AW, Huibregtse K, Tytgat GN. Four years experience with short term stenting in primary sclerosing cholangitis. Am J Gastroenterol 1999;94:2403-2407.

4. Burak K, Angulo P, Pasha TM, Egan K, Petz J, Lindor KD. Incidence and risk factors for cholangiocarcinoma in primary sclerosing cholangitis. Am J Gastroenterol 2004;99:523-526.

5. Claessen MMH, Vleggaar FP, Tytgat KM, Siersema PD, van Buuren HR. High lifetime risk of cancer in primary sclerosing cholangitis. J Hepatol 2009;50:158-164.

6. Patkowski W, Skalski M, Zieniewicz K, Nyckowski P, Smoter P, Krawczyk M. Orthotopic liver transplantation for cholestatic diseases. Hepatogastroenterology 2010;57:605-610.

7. Wiesner R, Grambsch P, Dickson E, Ludwig J, MacCarty RL, Hunter EB, et al. Primary sclerosing cholangitis: natural history, prognostic factors and survival analysis. Hepatology 1989;10:430-436.

8. Farrant JM, Hayllar KM, Wilkinson ML, Karani J, Portmann BC, Westaby D, Williams R. Natural history and prognostic variables in primary sclerosing cholangitis. Gastroenterology 1991;100:17101717.

9. Tischendorf JJW, Hecker H, Krüger M, Manns MP, Meier PN. Characterization, outcome, and prognosis in 273 patients with primary sclerosing cholangitis: a single center study. Am J Gastroenterol 2007;102: 107-114.

10. Lamberts LE, Janse M, Haagsma EB, van den Berg AP, Weersma RK. Immune-mediated diseases in primary sclerosing cholangitis. Dig Liver Dis 2011;43:802-806.

11. Janse M, Lamberts LE, Verdonk RC, Weersma RK. IBD is associated with an increase in carcinoma in PSC irrespective of the presence of dominant bile duct stenosis. J Hepatol 2012;57:473-474.

12. Metcalf J, James O. The geoepidemiology of primary biliary cirrhosis. Semin Liver Dis 1997;17:13-22.
13. Casparie M, Tiebosch A, Burger G, Blauwgeers H, van de Pol A, van Krieken JH, Meijer GA. Pathology databanking and biobanking in The Netherlands, a central role for PALGA, the nationwide histopathology and cytopathology data network and archive. Cell Oncol 2007;29:19-24.

14. EASL. EASL Clinical Practice Guidelines: management of cholestatic liver diseases. J Hepatol 2009;51:237-267.

15. Hennes EM, Zeniya M, Czaja AJ, Parés A, Dalekos GN, Krawitt EL, et al. Simplified criteria for the diagnosis of autoimmune hepatitis. Hepatology 2008;48:169-176.

16. Lennard-Jones J. Classification of inflammatory bowel disease. Scand J Gastroenterol 1989;170:2-6; discussion, 16-19.

17. Boonstra K, Beuers U, Ponsioen CY. Epidemiology of primary sclerosing cholangitis and primary biliary cirrhosis: a systematic review. J Hepatol 2012;56:1181-1188.

18. Boberg KM, Aadland E, Jahnsen J, Raknerud N, Stiris M, Bell H. Incidence and prevalence of primary biliary cirrhosis, primary sclerosing cholangitis, and autoimmune hepatitis in a Norwegian population. Scand J Gastroenterol 1998;33:99-103.

19. Bambha K, Kim R, Talwalkar J, Torgerson H, Benson JT, Therneau $\mathrm{TM}$, et al. Incidence, clinical spectrum, and outcomes of primary sclerosing cholangitis in a United States community. Gastroenterology 2003;125:1364-1369.

20. Kaplan GG, Laupland KB, Butzner D, Urbanski SJ, Lee SS. The burden of large and small duct primary sclerosing cholangitis in adults and children: a population-based analysis. Am J Gastroenterol 2007;102:1042-1049.

21. Vind I, Riis L, Jess T, Knudsen E, Pedersen N, Elkjaer M, et al. Increasing incidences of inflammatory bowel disease and decreasing surgery rates in Copenhagen City and County, 2003-2005: a populationbased study from the Danish Crohn colitis database. Am J Gastroenterol 2006;101:1274-1282.

22. Jussila A, Virta LJ, Kautiainen H, Rekiaro M, Nieminen U, Färkkilä MA. Increasing incidence of inflammatory bowel diseases between 2000 and 2007: a nationwide register study in Finland. Inflamm Bowel Dis 2012;18:555-561.

23. Hurlburt KJ, McMahon BJ, Deubner H, Hsu-Trawinski B, Williams JL, Kowdley KV. Prevalence of autoimmune liver disease in Alaska Natives. Am J Gastroenterol 2002;97:2402-2407.

24. Ngu JH, Gearry RB, Wright AJ, Stedman CA. Inflammatory bowel disease is associated with poor outcomes of patients with primary sclerosing cholangitis. Clin Gastroenterol Hepatol 2011;9:1092-1097.

25. Boberg KM, Bergquist A, Mitchell S, Pares A, Rosina F, Broomé U, et al. Cholangiocarcinoma in primary sclerosing cholangitis: risk factors and clinical presentation. Scand J Gastroenterol 2002;37:1205-1211.

26. Lindor K. Ursodiol for primary sclerosing cholangitis. $\mathrm{N}$ Engl J of Med 1997;336:691-695.

27. Olsson R, Boberg KM, de Muckadell OS, Lindgren S, Hultcrantz R, Folvik G, et al. High-dose ursodeoxycholic acid in primary sclerosing cholangitis: a 5-year multicenter, randomized, controlled study. Gastroenterology 2005;129:1464-1472.

28. Lindor K, Kowdley K, Luketic V, Harrison ME, McCashland T, Befeler AS, et al. High-dose ursodeoxycholic acid for the treatment of primary sclerosing cholangitis. HePATOLOGY 2009;50:808-814.

29. Mohammed N, El-Aleem S, El-Hafiz H, McMahon RF. Distribution of constitutive (COX-1) and inducible (COX-2) cyclooxygenase in postviral human liver cirrhosis: a possible role for COX-2 in the pathogenesis of liver cirrhosis. J Clin Pathol 2004;57:350-354.

30. Cervello M, Montalto G. Cyclooxygenases in hepatocellular carcinoma. World J Gastroenterol 2006;12:5113-5121.

31. Chávez E, Castro-Sánchez L, Shibayama M, Tsutsumi V, Pérez Salazar E, Moreno MG, Muriel P. Effects of acetyl salycilic acid and ibuprofen in chronic liver damage induced by CCl4. J Appl Toxicol 2012;32:51-59.

32. Elsharkawy AM, Mann DA. Nuclear factor kappa B and the hepatic inflammation-fibrosis-cancer axis. HePATOLOGY 2007;46:590-597.

33. Sahasrabuddhe V V, Gunja MZ, Graubard BI, Trabert B, Schwartz LM, Park Y, et al. Nonsteroidal anti-inflammatory drug use, chronic liver disease, and hepatocellular carcinoma. J Natl Cancer Inst 2012; 104:1808-1814 
34. Bergquist A, Ekbom A, Olsson R, Kornfeldt D, Löö L, Danielsson A, et al. Hepatic and extrahepatic malignancies and primary sclerosing cholangitis. J Hepatol 2002;36:321-327.

35. Tischendorf JJW, Meier PN, Strassburg CP, Klempnauer J, Hecker H, Manns MP, Krüger M, et al. Characterization and clinical course of hepatobiliary carcinoma in patients with primary sclerosing cholangitis. Scand J Gastroenterol 2006;41:1227-1234.

36. Herrinton LJ, Liu L, Levin TR, Allison JE, Lewis JD, Velayos F. Incidence and mortality of colorectal adenocarcinoma in persons with inflammatory bowel disease from 1998 to 2010. Gastroenterology 2012;143:382-389.

37. Jess T, Simonsen J, Jørgensen KT, Pedersen BV, Nielsen NM, Frisch M. Declining risk of colorectal cancer in patients with inflammatory bowel disease over 30 years. Gastroenterology 2012;143:375-381.
38. Broomé U, Lindberg G, Löfberg R. Primary sclerosing cholangitis in ulcerative colitis-a risk factor for the development of dysplasia and DNA aneuploidy? Gastroenterology 1992;102:1877-1880.

39. Torres J, Chambrun GP de, Itzkowitz S, Sachar DB, Colombel JF. Review article: colorectal neoplasia in patients with primary sclerosing cholangitis and inflammatory bowel disease. Aliment Pharmacol Ther 2011;34:497-508.

40. Valle MB de, Björnsson E, Lindkvist B. Mortality and cancer risk related to primary sclerosing cholangitis in a Swedish population-based cohort. Liver Int 2012;32:441-448.

41. Chapman R, Fevery J, Kalloo A, Nagorney DM, Boberg KM, Shneider B, Gores GJ; American Association for the Study of Liver Diseases. Diagnosis and management of primary sclerosing cholangitis. HepatoLOGY 2010;51:660-678. 